\title{
Author Correction: Stable pure-blue hyperfluorescence organic light-emitting diodes with high-efficiency and narrow emission
}

Chin-Yiu Chan (D), Masaki Tanaka (D), Yi-Ting Lee, Yiu-Wing Wong, Hajime Nakanotani (D), Takuji Hatakeyama (D) and

Chihaya Adachi (D)

Correction to: Nature Photonics https://doi.org/10.1038/s41566-020-00745-Z, published online 4 January 2021.

In the version of this Letter originally published, the labels of the light-blue and dark-blue lines in the legend of Fig. $1 \mathrm{~b}$ were the wrong way around; the light-blue line should have been labelled ' $0.5 \mathrm{wt} \% \nu$-DABNA: $20 \mathrm{wt} \% \mathrm{HDT}$-1:mCBP' and the dark-blue line should have

been labelled ' $0.5 \mathrm{wt} \% \nu$-DABNA: $m \mathrm{CBP}$ '. This has now been corrected in all versions of the Letter.

Published online: 12 January 2021

https://doi.org/10.1038/s41566-021-00759-1

(c) The Author(s), under exclusive licence to Springer Nature Limited 2021 